\title{
Current and predicted prevalence of obesity in Canada: a trend analysis
}

\author{
Laurie K. Twells PhD, Deborah M. Gregory PhD, Jacinta Reddigan MSc, William K. Midodzi PhD
}

\section{Abstract}

Background: The prevalence of obesity has increased over the past 3 decades, with a disproportionate growth in excessive weight categories (body mass index [BMI] 35.0-39.9 and BMI $\geq 40.0$ ). The objective of this paper is to present the data for the past and current prevalence of adult obesity in Canada, together with future estimates.

Methods: We calculated BMls for adults aged 18 years and older who were not in long-term care using data from Canadian health surveys administered between 1985 and 2011. Calculation of the BMls was based on self-reported heights and weights. The weight categories were as follows: normal (BMI 18.5-24.9), overweight (25.0-29.9), obese class I (30.0-34.9), obese class II (35.0-39.9) and obese class III ( $\geq 40.0)$. Outcome measures were prevalence of adult obesity according to BMI categories, nationally and provincially. We used regression analysis models to predict future prevalence of adult obesity up to 2019.

Results: Between 1985 and 2011, the prevalence of adult obesity in Canada increased from 6.1\% to 18.3\%. Furthermore, since 1985, the prevalence of obesity in classes I, II and III increased from $5.1 \%$ to $13.1 \%$, from $0.8 \%$ to $3.6 \%$, and from $0.3 \%$ to $1.6 \%$, respectively. Taking into account regional variations, we predict that, by 2019 , the prevalence of obesity in classes I, II and III will increase to $14.8 \%, 4.4 \%$ and $2.0 \%$, respectively, and that half of the Canadian provinces will have more overweight or obese adults than normalweight adults.

Interpretation: We found significant increases in the excessive weight categories of obesity, with continued increases predicted for all provinces up to 2019. Provincial variations in obesity prevalence were also significant. To address these projected increases and any subsequent burden on the health care system, a concerted effort must be made by the provinces to focus on the prevention, management and treatment of obesity in Canada.

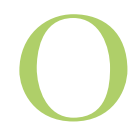

ver almost the past 3 decades, the prevalence of obesity, defined by a body mass index (BMI) of 30 or higher, has increased in many parts of the world. In Canada, the prevalence of obesity began to increase steadily in the 1980s. According to the 1985 Canadian Health Promotion Survey, about $6.1 \%$ of Canadian adults were found to be obese, compared with $18.1 \%$ reported from the findings of the 2010 Canadian Community Health Survey (CCHS)..$^{1-3}$ These national figures mask the substantial provincial variations that exist. ${ }^{4,5}$

Obese people are at an increased risk of hypertension, type 2 diabetes, sleep apnea and cancer. ${ }^{6-8}$ Excess body weight can also affect quality of life, education and income potential, ${ }^{9-11}$ and increase the risk of premature death. ${ }^{12,13}$ Obesity is often categorized by BMI to reflect the increasing health risk of excess weight: class I (BMI 30.0-34.9), class II (BMI 35.0$39.9)$ and class III (BMI $\geq 40.0)$. ${ }^{14,15}$ The latter 2 categories are linked to much higher levels of morbidity, increased demands on the health care system and premature death..$^{16-19}$ There has been a disproportionate increase in people within obesity classes II and III in Canada. ${ }^{2,20}$ Taking into account figures for the past 2 decades, the annual economic burden of obesity is now estimated to be between $\$ 4.6$ billion and $\$ 7.1$ billion, ${ }^{21,22}$ and it has been suggested that obesity contributes as much as, if not more than, smoking to the onset of disease. ${ }^{23}$

The purpose of this paper is to present the data for the past (10 yr) and current prevalence of adult obesity in Canada, together with future estimates. These data are important in monitoring and evaluating the effectiveness of populationand community-wide interventions aimed at reducing obesity, as well as in estimating the future burden on the health care system of disease associated with excess body weight.

Competing interests: None declared.

This article has been peer reviewed.

Correspondence to: Laurie K. Twells, Itwells@mun.ca

CMAJ Open 2014.DOI:10.9778/cmajo.20130016 


\section{Methods}

\section{Setting}

We analyzed BMI data from cross-sectional Canadian health surveys administered to adults aged 18 years and older who were not in long-term care. In the first CCHS, conducted by Statistics Canada in 2000/01, only adults aged 20-64 years participated in the study. For subsequent surveys (i.e., 2003 onwards), adults were defined as aged 18 years and older.

\section{Data sources}

To examine the national obesity rates before 2000, we used data published from the 1985 and 1990 cross-sectional Health Promotion Surveys and the longitudinal 1994/95, 1996/97 and 1998/99 National Population Health Surveys. ${ }^{2}$ The 2000/01-2011 study sample was obtained from the cross-sectional CCHSs. For our analysis, we used data from the CCHS cycles 1.1 (2001), 2.1 (2003), 3.1 (2005) and 4.1 (2007) and from the 2007/08, 2009/10 and 2011 CCHSs. Full details of the study design and procedures have been reported previously. ${ }^{24-26}$ Because few data were reported from the Canadian territories, we did not include these data in the current analysis. Given the focus of the paper, we also did not include data from the underweight category.

\section{Outcome measures}

Body mass index values are categorized as follows: normal weight (BMI 18.5-24.9), overweight (BMI 25.0-29.9), obese class I (BMI 30.0-34.9), obese class II (BMI 35.0-39.9) and obese class III (BMI $\geq 40.0) .^{14,15}$ In our study, BMI was calculated using selfreported heights and weights gathered from the 3 surveys.

\section{Statistical analysis}

National trends are presented from 1985 to 2011 and provincial trends from 2000/01 to 2011. We conducted subgroup analyses for national and provincial data, and for BMI category, by age and sex, and included percentage changes over time between 2000/01 and 2011. The interpretation of data on obesity prevalence by sex and age is limited for obese classes II and III owing to high sampling variability and the lack of data in the oldest age category ( $\geq 60 \mathrm{yr}$ ) for the $2000 / 01 \mathrm{CCHS}$. Some data points must be interpreted with caution, especially in the subgroup analysis. Such points are indicated in the tables. We estimated national and provincial prevalence rates for BMI categories to 2019. All surveys were weighted to be representative of both national and provincial populations. The 10 -year historical BMI prevalence data between 2000/01 and 2011 were tested for linear, quadratic and cubic trends. Based on visual inspection and evaluation of the models, we chose a linear model as the best fit for the data and used it in the prediction analysis. The trend analysis was limited to 6 data points over a 10-year period, because the CCHS was only started in 2000. As a result, we used linear regression to estimate the future prevalence of obesity up to $2019 .{ }^{27}$ This linear line technique has been used frequently to describe population prevalence or growth, and to make predictions for the future. We performed data analyses using IBM SPSS Version 20.

\section{Results}

\section{National results, 1985-2011}

Between 1985 and 2011, the prevalence of adults in the overweight category increased by $21 \%$ from $27.8 \%$ to $33.6 \%$, and the prevalence of obesity (BMI $\geq 30.0$ ) increased $200 \%$ from $6.1 \%$ to $18.3 \%$. All classes of obesity increased over this period, with disproportionate increases seen in the higher obese classes. The prevalence for obese class I increased from $5.1 \%$ to $13.1 \%$, obese class II from $0.8 \%$ to $3.6 \%$, and obese class III from $0.3 \%$ to $1.6 \%$ (Figure 1 ).

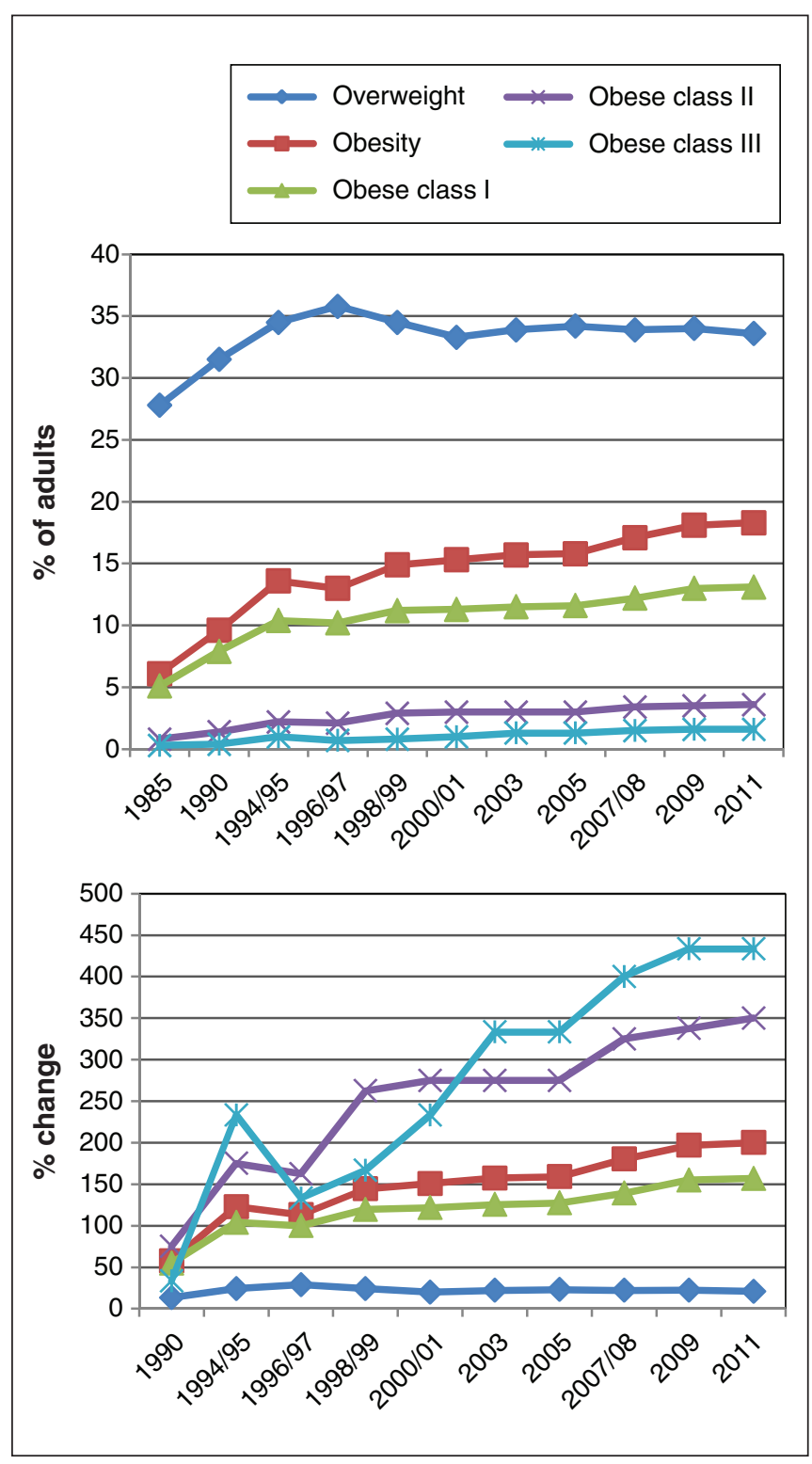

Figure 1: (A) Prevalence of adults classified as overweight or obese in Canada from 1985 to 2011, by weight category: overweight (BMI 25.0-29.9), obese (BMI $\geq 30.0$ ), obese class I (BMI 30.0-34.9), obese class II (BMI 35.0-39.9) and obese class III (BMI $\geq 40.0$ ). (B) Percentage change in the prevalence of overweight and obesity in Canada, by weight category, using 1985 data as a baseline. $\mathrm{BMI}=$ body mass index. 


\section{OPEN}

\section{Provincial results, 2000/01-2011}

Between 2000/01 and 2011, the prevalence of overweight decreased in Prince Edward Island, New Brunswick and Alberta, whereas there were increases in the other provinces. All provinces had increases in obese class I, II (except for Saskatchewan) and III. Lower prevalence rates of obesity were observed in the west and higher rates in the east. In 2011, the prevalence of overweight varied from $31.3 \%$ in British Columbia to $41.8 \%$ in Newfoundland and Labrador. The rate for obese class I varied from $10.7 \%$ in BC to $20.5 \%$ in Newfoundland and Labrador. For obese class II, New Brunswick reported the highest rate $(6.1 \%)$ and Quebec the lowest $(2.5 \%)$. For obese class III, New Brunswick reported the highest rate $(2.8 \%)$, and $\mathrm{BC}$ and Quebec the lowest at $1.2 \%$ (Table 1). Appendix 1, available at www.cmaj open.ca/content/2/1/E18/suppl/DC1, shows the prevalence of BMI categories as reported by the CCHS for 2000/01 to 2011.

\section{Subgroup analysis}

\section{BMI categories by sex and age}

Table 2 shows the prevalence for the overweight and obese BMI categories by sex for 2000/01 and 2011, as well as national and provincial percentage changes. For women, increases in the overweight category were reported for most provinces (ranging from an increase of $3.8 \%$ in Ontario to an increase of $18.0 \%$ in Nova Scotia), with the exception of Prince Edward Island and Alberta, which had decreases of $24.5 \%$ and $6.1 \%$, respectively. For overweight men, increases were reported in 6 of 10 provinces, ranging from $1.0 \%$ in Nova Scotia to $5.2 \%$ in Newfoundland and Labrador. Prince Edward Island, New Brunswick, Ontario and Alberta all had decreases in the prevalence of men in the overweight category, ranging from $0.5 \%$ in Alberta to $6.6 \%$ in New Brunswick. In both survey years, men were more likely to be overweight than women, a finding that was consistent across all provinces.

Table 1: Provincial and national prevalence (\%) of body mass index categories in adults ( $\geq 18$ yr) and percentage changes for $2000 / 01$ and 2011

\begin{tabular}{|c|c|c|c|c|c|c|c|c|c|c|c|}
\hline \multirow{2}{*}{$\begin{array}{l}\text { BMI category, }{ }^{*}- \\
\text { year }\end{array}$} & \multicolumn{10}{|c|}{ Provincial } & \multirow[b]{2}{*}{ National } \\
\hline & NL & PEI & NS & NB & QC & ON & MB & SK & $A B$ & BC & \\
\hline \multicolumn{12}{|l|}{ Normal } \\
\hline $2000 / 01$ & 39.0 & 40.5 & 42.1 & 41.9 & 52.4 & 47.9 & 45.5 & 43.0 & 47.6 & 53.8 & 49.0 \\
\hline 2011 & 29.9 & 41.8 & 37.0 & 38.7 & 47.1 & 45.4 & 40.0 & 39.0 & 45.3 & 51.4 & 45.6 \\
\hline$\%$ change & -23.3 & 3.2 & -12.1 & -7.6 & -10.1 & -5.2 & -12.1 & -9.3 & -4.8 & -4.5 & -6.9 \\
\hline \multicolumn{12}{|l|}{ Overweight } \\
\hline $2000 / 01$ & 38.2 & 38.9 & 34.9 & 35.0 & 31.9 & 33.6 & 34.7 & 35.0 & 33.9 & 30.7 & 33.1 \\
\hline 2011 & 41.8 & 34.3 & 37.5 & 34.3 & 33.3 & 33.7 & 36.5 & 37.3 & 33.0 & 31.3 & 33.6 \\
\hline$\%$ change & 9.4 & -11.8 & 7.4 & -2.0 & 4.4 & 0.3 & 5.2 & 6.6 & -2.7 & 2.0 & 1.5 \\
\hline \multicolumn{12}{|l|}{ Obese } \\
\hline $2000 / 01$ & 21.7 & 19.6 & 21.4 & 21.4 & 12.7 & 15.6 & 18.4 & 20.2 & 16.4 & 12.5 & 15.2 \\
\hline 2011 & 27.7 & 23.5 & 23.7 & 25.1 & 16.8 & 18.4 & 21.6 & 22.1 & 19.3 & 14.5 & 18.3 \\
\hline$\%$ change & 27.6 & 19.9 & 10.7 & 17.3 & 32.3 & 17.9 & 17.4 & 9.4 & 17.7 & 16.0 & 20.4 \\
\hline \multicolumn{12}{|l|}{ Obese class I } \\
\hline $2000 / 01$ & 16.1 & 14.7 & 14.7 & 16.0 & 9.5 & 11.5 & 13.0 & 14.4 & 12.2 & 9.3 & 11.3 \\
\hline 2011 & 20.5 & 18.0 & 15.6 & 16.2 & 13.1 & 12.8 & 15.2 & 15.9 & 13.5 & 10.7 & 13.1 \\
\hline$\%$ change & 27.3 & 22.4 & 6.1 & 1.3 & 37.9 & 11.3 & 16.9 & 10.4 & 10.7 & 15.1 & 15.9 \\
\hline \multicolumn{12}{|l|}{ Obese class II } \\
\hline $2000 / 01$ & 4.1 & 3.3 & 4.8 & 3.7 & 2.4 & 2.9 & 3.8 & 4.3 & 2.9 & 2.2 & 2.8 \\
\hline 2011 & 4.6 & 3.6 & 5.8 & 6.1 & 2.5 & 4.0 & 4.0 & 4.3 & 4.3 & 2.6 & 3.6 \\
\hline$\%$ change & 12.2 & 9.1 & 20.8 & 64.9 & 4.2 & 37.9 & 5.3 & 0.0 & 48.3 & 18.2 & 28.6 \\
\hline \multicolumn{12}{|l|}{ Obese class III } \\
\hline $2000 / 01$ & 1.5 & 1.6 & 1.9 & 1.8 & 0.8 & 1.2 & 1.7 & 1.5 & 1.2 & 0.9 & 1.2 \\
\hline 2011 & 2.6 & 2.5 & 2.3 & 2.8 & 1.2 & 1.7 & 2.4 & 2.0 & 1.4 & 1.2 & 1.6 \\
\hline$\%$ change & 73.3 & 56.3 & 21.1 & 55.6 & 50.0 & 41.7 & 41.2 & 33.3 & 16.7 & 33.3 & 33.3 \\
\hline $\begin{array}{l}\text { Note: BMI = body ma } \\
\text { Data source: Statisti } \\
\text { Newfoundland and L } \\
\text { "BMI categories: nor } \\
\text { III (BMI } \geq 40.0 \text { ). }\end{array}$ & $\begin{array}{l}\text { ass index } \\
\text { cs Canad } \\
\text {-abrador } \\
\text { mal (BMI }\end{array}$ & $\begin{array}{l}\text { anadian } \\
\text { e for } \mathrm{He} \\
-24.9) \text {, }\end{array}$ & $\begin{array}{l}\text { munity } \mathrm{H} \\
\text { Informatic } \\
\text { weight (B }\end{array}$ & $\begin{array}{r}\text { Surve } \\
50-29 .\end{array}$ & $\begin{array}{l}\text { re File, } \\
\text { ese (BN }\end{array}$ & ), obe & $\begin{array}{l}\text { ), } 2003 \\
\text { I (BMI }\end{array}$ & $\begin{array}{l}2007 \\
34.9),\end{array}$ & $\begin{array}{l}\text { 2009-2 } \\
\text { lass II ( }\end{array}$ & $\begin{array}{l}\text { d } 2011 \\
.0-39.9\end{array}$ & $\begin{array}{l}\text { led by the } \\
\text { bese class }\end{array}$ \\
\hline
\end{tabular}


Between 2000/01 and 2011, increases in the prevalence rate for the obese category ( $\mathrm{BMI} \geq 30.0$ ) were reported for both women and men for all provinces. Across all provinces, there was a higher reported prevalence of men in the obese categories. Between 2000/01 and 2011, increases in prevalence were reported for women in obese class I for all provinces, except for New Brunswick and Saskatchewan, where the prevalence decreased by $1.3 \%$ and $3.1 \%$, respectively. For men, an increased prevalence in obese class I was reported across all provinces. Overall, men were more likely than women to be categorized as obese class I. It is difficult to draw conclusions for the higher classes of obesity, because the data must be interpreted with caution; however, these data illustrate increases in the prevalence of both men and women in obese

Table 2 (part 1 of 2): National and provincial prevalence (\%) of body mass index categories in adults ( $\geq 18 \mathrm{yr})$ and percentage changes for $2000 / 01$ and 2011 , by sex

Provincial

\begin{tabular}{|c|c|c|c|c|c|c|c|c|c|c|c|}
\hline \multirow{2}{*}{$\begin{array}{l}\text { BMI category, } \\
\text { year, sex }\end{array}$} & \multicolumn{10}{|c|}{ Provincial } & \multirow[b]{2}{*}{ National } \\
\hline & NL & PEI & NS & NB & QC & ON & MB & SK & $A B$ & BC & \\
\hline \multicolumn{12}{|l|}{ Normal } \\
\hline \multicolumn{12}{|l|}{$2000 / 01$} \\
\hline Women & 45.1 & 43.7 & 49.8 & 47.1 & 58.1 & 54.6 & 40.6 & 50.7 & 55.0 & 60.1 & 55.4 \\
\hline Men & 33.0 & 33.7 & 34.6 & 36.9 & 46.9 & 41.5 & 50.8 & 35.8 & 40.9 & 47.7 & 42.9 \\
\hline \multicolumn{12}{|l|}{2011} \\
\hline Women & 36.8 & 52.7 & 41.9 & 41.5 & 53.0 & 51.4 & 46.0 & 45.9 & 54.5 & 57.4 & 51.8 \\
\hline Men & 22.9 & 30.9 & 31.8 & 35.9 & 41.2 & 39.2 & 34.0 & 32.3 & 36.8 & 45.4 & 39.3 \\
\hline$\%$ change women & -18.4 & 20.6 & -15.9 & -11.9 & -8.8 & -5.9 & 13.3 & -9.5 & -0.9 & -4.5 & -6.5 \\
\hline$\%$ change men & -30.6 & -8.3 & -8.1 & -2.7 & -12.2 & -5.5 & -33.1 & -9.8 & -10.0 & -4.8 & -8.4 \\
\hline \multicolumn{12}{|l|}{ Overweight } \\
\hline \multicolumn{12}{|l|}{ 2000/01 } \\
\hline Women & 32.1 & 33.5 & 28.4 & 29.0 & 24.8 & 26.6 & 28.6 & 27.4 & 26.4 & 22.8 & 26.0 \\
\hline Men & 44.1 & 44.0 & 41.4 & 40.7 & 38.7 & 40.3 & 40.4 & 41.9 & 40.7 & 38.2 & 39.9 \\
\hline \multicolumn{12}{|l|}{2011} \\
\hline Women & 37.2 & 25.3 & 33.5 & 30.8 & 26.6 & 27.6 & 30.5 & 30.7 & 24.8 & 23.7 & 27.1 \\
\hline Men & 46.4 & 43.3 & 41.8 & 38.0 & 39.9 & 39.9 & 42.4 & 43.8 & 40.5 & 38.9 & 40.1 \\
\hline$\%$ change women & 15.9 & -24.5 & 18.0 & 6.2 & 7.3 & 3.8 & 6.6 & 12.0 & -6.1 & 3.9 & 4.2 \\
\hline$\%$ change men & 5.2 & -1.6 & 1.0 & -6.6 & 3.1 & -1.0 & 5.0 & 4.5 & -0.5 & 1.8 & 0.5 \\
\hline \multicolumn{12}{|l|}{ Obese } \\
\hline \multicolumn{12}{|l|}{$2000 / 01$} \\
\hline Women & 21.1 & 17.1 & 19.7 & 21.6 & 12.1 & 14.5 & 18.1 & 18.7 & 15.2 & 11.9 & 14.4 \\
\hline Men & 22.2 & 21.9 & 23.1 & 21.3 & 13.3 & 16.8 & 18.6 & 21.5 & 17.4 & 13.0 & 16.1 \\
\hline \multicolumn{12}{|l|}{2011} \\
\hline Women & 25.3 & 21.5 & 21.8 & 25.0 & 15.7 & 17.3 & 21.2 & 20.8 & 16.7 & 13.9 & 17.1 \\
\hline Men & 30.2 & 25.7 & 25.8 & 25.3 & 17.9 & 19.7 & 22.1 & 23.4 & 21.7 & 15.0 & 19.5 \\
\hline$\%$ change women & 19.9 & 25.7 & 10.7 & 15.7 & 29.8 & 19.3 & 17.1 & 11.2 & 9.9 & 16.8 & 18.8 \\
\hline$\%$ change men & 36.0 & 17.4 & 11.7 & 18.8 & 34.6 & 17.3 & 18.8 & 8.8 & 24.7 & 15.4 & 21.1 \\
\hline \multicolumn{12}{|l|}{ Obese class I } \\
\hline \multicolumn{12}{|l|}{$2000 / 01$} \\
\hline Women & 14.2 & 12.1 & 12.9 & 15.6 & 8.4 & 10.0 & 11.6 & 13.1 & 10.5 & 8.2 & 9.9 \\
\hline Men & 17.9 & 17.2 & 16.4 & 16.3 & 10.5 & 13.1 & 14.2 & 15.6 & 12.9 & 10.4 & 12.6 \\
\hline \multicolumn{12}{|l|}{2011} \\
\hline Women & 17.0 & 16.4 & 13.8 & 15.4 & 11.4 & 10.9 & 14.3 & 12.7 & 11.0 & 9.7 & 11.3 \\
\hline Men & 24.2 & 19.6 & 17.6 & 17.0 & 14.9 & 14.8 & 16.2 & 18.9 & 15.8 & 11.7 & 15.0 \\
\hline$\%$ change women & 19.7 & 35.5 & 7.0 & -1.3 & 35.7 & 9.0 & 23.3 & -3.1 & 4.8 & 18.3 & 14.1 \\
\hline$\%$ change men & 35.2 & 14.0 & 7.3 & 4.3 & 41.9 & 13.0 & 14.1 & 21.2 & 22.5 & 12.5 & 19.0 \\
\hline
\end{tabular}




\section{OPEN}

Research

\begin{tabular}{|c|c|c|c|c|c|c|c|c|c|c|c|}
\hline \multirow{2}{*}{$\begin{array}{l}\text { BMI category, } \\
\text { year, sex }\end{array}$} & \multicolumn{10}{|c|}{ Provincial } & \multirow[b]{2}{*}{ National } \\
\hline & NL & PEI & NS & NB & $Q C$ & ON & MB & SK & $A B$ & $\mathrm{BC}$ & \\
\hline \multicolumn{12}{|l|}{ Obese class II } \\
\hline \multicolumn{12}{|l|}{$2000 / 01$} \\
\hline Women & 4.7 & $3.4 \dagger$ & 4.4 & 3.7 & 2.7 & 2.9 & 4.2 & 3.9 & 3.0 & 2.6 & 3.0 \\
\hline Men & $3.5 \dagger$ & $3.2 \dagger$ & 5.2 & 3.7 & 2.1 & 2.8 & $3.3 \dagger$ & 4.7 & 2.9 & 1.8 & 2.7 \\
\hline \multicolumn{12}{|l|}{2011} \\
\hline Women & $5.2 \dagger$ & - & 5.8 & $5.8 \dagger$ & 2.8 & 4.2 & $4.0 \dagger$ & $6.2 \dagger$ & 3.9 & 2.5 & 3.8 \\
\hline Men & $4.0 \dagger$ & 一‡ & $5.8 \dagger$ & $6.5 \dagger$ & 2.1 & 3.7 & $4.0 \dagger$ & $2.5 \dagger$ & $4.5 \dagger$ & $2.7 \dagger$ & 3.4 \\
\hline$\%$ change women & 10.6 & 一‡ & 31.8 & 56.8 & 3.7 & 44.8 & -4.8 & 59.0 & 30.0 & -3.9 & 26.67 \\
\hline$\%$ change men & 14.3 & -‡ & 11.5 & 75.7 & 0.0 & 32.1 & 21.2 & -46.8 & 55.2 & 50.0 & 25.9 \\
\hline \multicolumn{12}{|l|}{ Obese class III } \\
\hline \multicolumn{12}{|l|}{$2000 / 01$} \\
\hline Women & $2.2 \dagger$ & -‡ & 2.3 & $2.3 \dagger$ & 1 & 1.6 & $2.3 \dagger$ & 1.8 & 1.8 & 1.1 & 1.5 \\
\hline Men & -‡ & $1.5 \dagger$ & $1.4 \dagger$ & $1.3 \dagger$ & $0.7 \dagger$ & 0.9 & $1.1 \dagger$ & $1.3 \dagger$ & $0.7 \dagger$ & $0.8 \dagger$ & 0.9 \\
\hline \multicolumn{12}{|l|}{2011} \\
\hline Women & $3 \dagger$ & $-\ddagger$ & $2.2 \dagger$ & $3.8 \dagger$ & 1.4 & 2.1 & $2.9 \dagger$ & $1.9 \dagger$ & $1.8 \dagger$ & $1.7 \dagger$ & 1.9 \\
\hline Men & - & -‡ & $2.5 \dagger$ & - & $1.0 \dagger$ & 1.2 & $1.9 \dagger$ & $2.0 \dagger$ & $1.1 \dagger$ & -‡ & 1.2 \\
\hline$\%$ change women & $36.4 \dagger$ & -‡ & $-4.3 \dagger$ & $65.2 \dagger$ & 40.0 & 31.3 & $26.1 \dagger$ & $5.6 \dagger$ & $0.0 \dagger$ & $54.5 \dagger$ & 26.7 \\
\hline$\%$ change men & - & 一‡ & $78.6 \dagger$ & 一 & $42.9 \dagger$ & 33.3 & $72.7 \dagger$ & $53.8 \dagger$ & $57.1 \dagger$ & -‡ & 33.3 \\
\hline $\begin{array}{l}\text { Note: BMI = body mas } \\
\text { Data source: Statistics } \\
\text { and older (compiled by } \\
\text { *BMI categories: norm } \\
\text { III (BMI } \geq 40.0 \text { ). } \\
\text { †Data with a coefficien } \\
\text { fData suppressed bec }\end{array}$ & $\begin{array}{l}\text { Idex. } \\
\text { Inada, C } \\
\text { e Newfol } \\
\text { BMl } 18.5\end{array}$ & id anc & $\begin{array}{l}\text { y Health } \\
\text { dor, Cent } \\
\text { (BMI } 25\end{array}$ & $\begin{array}{l}\text { urvey, She } \\
\text { for Healt1 } \\
\text {-29.9), ob }\end{array}$ & $\begin{array}{l}\text { le, 2000/0 } \\
\text { rmation). } \\
\text { (BMI } \geq 30 \text {. }\end{array}$ & $\begin{array}{l}20-64 \mathrm{y} \\
\text { obese c }\end{array}$ & $\begin{array}{l}2003,20 \\
\text { (BMI } 30 .\end{array}$ & $\begin{array}{l}\text {,2007-200 } \\
\text { 34.9), obes }\end{array}$ & $\begin{array}{l}\text { 009-2010 } \\
\text { ass II (BM }\end{array}$ & $\begin{array}{l}\text { nd } 2011 \text { for } \\
.0-39.9 \text { ) a }\end{array}$ & $\begin{array}{l}\text { ge } 18 \text { years } \\
\text { l obese class }\end{array}$ \\
\hline
\end{tabular}

classes II and III. Women were more likely than men to be classified as obese class II and III.

Table 3 shows the prevalence for the BMI categories by age (18-39, 40-59 and $\geq 60 \mathrm{yr}$ ) for 2000/01 and 2011, showing both national and provincial percentage changes. There was a higher prevalence for the overweight and obese class I categories in the older age categories (40-59 and $\geq 60 \mathrm{yr}$ ) compared with the younger group. Subgroup analysis of the prevalence for obese classes II and III was limited owing to unreliable data.

\section{Prediction analysis}

\section{National trends}

By 2019, we estimate that most (55.4\%) of the Canadian adult population will be categorized as overweight (34.2\%) or obese (21.2\%). Furthermore, we estimate that the prevalence for obese classes I, II and III will increase to $14.8 \%, 4.4 \%$ and $2.0 \%$, respectively (Figure 2 ).

\section{Provincial trends}

Appendix 2, available at www.cmajopen.ca/content/2/1 /E18/suppl/DC1, shows the predicted prevalence for all BMI categories and all provinces. By 2019, an estimated $71 \%$ of the adult population in Newfoundland and Labrador will be over- weight or obese. In Nova Scotia, New Brunswick, Saskatchewan and Manitoba, we estimate that there will be more overweight than normal-weight adults. In these provinces, the prevalence of adults in the overweight category will range from an estimated $34 \%$ to $38.5 \%$, and the prevalence of adults categorized as obese will range from an estimated $25.0 \%$ to $30.7 \%$. We estimate that Prince Edward Island, Ontario, Quebec, Alberta and BC will continue to have a lower prevalence for the overweight category (31.2\%-34.6\%) and obese category (15.7\%-26.0\%). Despite provincial variability, we predict increased prevalence for all obese classes in all provinces. Higher rates are predicted for eastern Canada, and lower rates for western Canada. Appendix 3, available at www.cmajopen.ca/content/2/1/E18/suppl/DC1, shows the regression analysis.

\section{Interpretation}

The results from our analysis show that the prevalence of normal-weight people in Canada is steadily decreasing, whereas the prevalence of all classes of obesity continues to rise, with the greatest percentage increases occurring in the excessive weight classes. In 2011, about $18.3 \%$ of adults in Canada were considered obese, equating to more than 4.8 million people with a BMI of 30.0 or greater. ${ }^{28}$ It is predicted that obesity rates will continue 
to rise in all provinces up to 2019. Our data show significant provincial variations in the prevalence and rate of change of obesity levels over time. For example, increases in the prevalence for obese class III were reported for all provinces; however, Newfoundland and Labrador showed a much higher rate of increase (73.3\%) compared with the other provinces. Although subgroup analysis by sex and age was limited, the data suggested that the prevalence for obesity in both men and women had increased over the past decade, with men more likely to be categorized as obese class I, and women as obese class II or class III. With a few exceptions, the prevalence of overweight and obesity was higher in the older age groups (40-59 and $\geq 60 \mathrm{yr}$ ) than that for the younger age group (18-39 yr) in all provinces.

Similar to many countries, Canada has experienced a substantial increase in the prevalence of obesity. ${ }^{1,29-31} \mathrm{~A}$ report by the Organisation for Economic Co-operation and Development

Table 3 (part 1 of 2): National and provincial prevalence (\%) of body mass index categories in adults ( $\geq 18$ yr), and percentage changes for 2000/01 and 2011, by age (yr)

\section{Provincial}

\begin{tabular}{|c|c|c|c|c|c|c|c|c|c|c|c|}
\hline \multirow{2}{*}{$\begin{array}{l}\text { BMI category, } \\
\text { year, age }\end{array}$} & & \multirow[b]{2}{*}{ Nationa } \\
\hline & NL & PEI & NS & NB & QC & ON & MB & SK & $A B$ & BC & \\
\hline \multicolumn{12}{|l|}{ Normal } \\
\hline \multicolumn{12}{|l|}{$2000 / 2001$} \\
\hline $18-39$ & 44.3 & 46.2 & 49.2 & 47.8 & 59.9 & 54.6 & 52.4 & 50.0 & 53.4 & 58.5 & 55.4 \\
\hline $40-59$ & 33.7 & 36.3 & 37.0 & 37.6 & 47.2 & 42.3 & 39.9 & 38.1 & 42.6 & 50.2 & 44.0 \\
\hline$\geq 60$ & NA & NA & NA & NA & NA & NA & NA & NA & NA & NA & NA \\
\hline \multicolumn{12}{|l|}{2011} \\
\hline $18-39$ & 32.3 & 58.9 & 46.5 & 46.9 & 56.1 & 53.5 & 47.0 & 47.2 & 52.6 & 60.5 & 54.0 \\
\hline $40-59$ & 28.1 & 34.9 & 31.3 & 35.8 & 42.5 & 41.2 & 37.1 & 34.6 & 39.4 & 47.4 & 41.2 \\
\hline$\geq 60$ & 30.0 & 33.0 & 34.2 & 33.1 & 41.5 & 38.9 & 34.2 & 33.2 & 40.3 & 44.0 & 39.6 \\
\hline$\%$ change 18-39 & -27.1 & 27.5 & -5.5 & -1.9 & -6.3 & -2.0 & -10.3 & -5.6 & -1.5 & 3.4 & -2.5 \\
\hline$\%$ change $40-59$ & -16.6 & -3.9 & -15.4 & -4.8 & -10.0 & -2.6 & -7.0 & -9.2 & -7.5 & -5.6 & -6.4 \\
\hline$\%$ change $\geq 60$ & NA & NA & NA & NA & NA & NA & NA & NA & NA & NA & NA \\
\hline \multicolumn{12}{|l|}{ Overweight } \\
\hline \multicolumn{12}{|l|}{$2000 / 2001$} \\
\hline $18-39$ & 33.9 & 35.5 & 30.6 & 32.5 & 25.7 & 28.8 & 30.0 & 31.7 & 29.5 & 26.7 & 28.3 \\
\hline $40-59$ & 42.6 & 41.1 & 37.7 & 36.5 & 36.2 & 37.8 & 38.3 & 37.3 & 37.1 & 33.7 & 36.8 \\
\hline$\geq 60$ & NA & NA & NA & NA & NA & NA & NA & NA & NA & NA & NA \\
\hline \multicolumn{12}{|l|}{2011} \\
\hline 18-39 & 34.7 & 23.8 & 34.2 & 27.7 & 39.5 & 41.8 & 48.7 & 50.9 & 43.5 & 35.0 & 41.8 \\
\hline $40-59$ & 45.7 & 37.3 & 37.5 & 34.9 & 55.6 & 57.7 & 62.5 & 63.9 & 59.5 & 50.8 & 57.5 \\
\hline$\geq 60$ & 44.1 & 41.7 & 41.5 & 41.6 & 56.5 & 59.8 & 65.3 & 65.6 & 58.0 & 54.1 & 58.9 \\
\hline$\%$ change 18-39 & 2.4 & -33.0 & 11.8 & -14.8 & 53.7 & 45.1 & 62.3 & 60.6 & 47.5 & 31.1 & 47.7 \\
\hline$\%$ change $40-59$ & 7.3 & -9.2 & -0.5 & -4.4 & 53.6 & 52.6 & 63.2 & 71.3 & 60.4 & 50.7 & 56.3 \\
\hline$\%$ change $\geq 60$ & NA & NA & NA & NA & NA & NA & NA & NA & NA & NA & NA \\
\hline \multicolumn{12}{|l|}{ Obese } \\
\hline \multicolumn{12}{|l|}{$2000 / 2001$} \\
\hline $18-39$ & 20.1 & 16.5 & 17.8 & 17.6 & 10.5 & 12.6 & 15.9 & 15.0 & 13.8 & 10.1 & 12.5 \\
\hline $40-59$ & 23.0 & 22.3 & 24.5 & 24.6 & 14.5 & 18.2 & 20.9 & 23.8 & 19.3 & 14.3 & 17.8 \\
\hline$\geq 60$ & NA & NA & NA & NA & NA & NA & NA & NA & NA & NA & NA \\
\hline \multicolumn{12}{|l|}{2011} \\
\hline $18-39$ & 32.1 & $17.1 \dagger$ & 16.2 & 21.6 & 13.4 & 14.1 & 16.6 & 17.7 & 15.3 & 10.8 & 14.3 \\
\hline $40-59$ & 25.9 & 27.4 & 30.8 & 28.0 & 19.4 & 22.1 & 27.5 & 25.5 & 22.9 & 17.2 & 21.7 \\
\hline$\geq 60$ & 25.5 & 25.0 & 22.5 & 25.0 & 17.8 & 19.6 & 20.8 & 23.8 & 21.1 & 15.6 & 19.3 \\
\hline$\%$ change 18-39 & 59.7 & $3.6 \% \dagger$ & -9.0 & 22.7 & 27.6 & 11.9 & 4.4 & 18.0 & 10.9 & 6.9 & 14.4 \\
\hline$\%$ change $40-59$ & 12.6 & 22.9 & 25.7 & 13.8 & 33.8 & 21.4 & 31.6 & 7.1 & 18.7 & 20.3 & 21.9 \\
\hline$\%$ change $\geq 60$ & NA & NA & NA & NA & NA & NA & NA & NA & NA & NA & NA \\
\hline
\end{tabular}


Table 3 (part 2 of 2): National and provincial prevalence (\%) of body mass index categories in adults ( $\geq 18$ yr), and percentage changes for $2000 / 01$ and 2011 , by age (yr)

\section{Provincial}

\begin{tabular}{|c|c|c|c|c|c|c|c|c|c|c|c|}
\hline \multirow{2}{*}{$\begin{array}{l}\text { BMI category, } \\
\text { year, age }\end{array}$} & & \multirow[b]{2}{*}{ National } \\
\hline & NL & PEI & NS & NB & $Q C$ & ON & MB & SK & AB & BC & \\
\hline \multicolumn{12}{|l|}{ Obese class I } \\
\hline \multicolumn{12}{|l|}{$2000 / 2001$} \\
\hline $18-39$ & 14.3 & 11.6 & 11.8 & 12.4 & 7.7 & 9.3 & 11.9 & 10.2 & 9.9 & 7.7 & 9.2 \\
\hline $40-59$ & 17.6 & 17.1 & 17.4 & 18.6 & 11.0 & 13.4 & 13.9 & 17.1 & 14.9 & 10.6 & 13.0 \\
\hline$\geq 60$ & NA & NA & NA & NA & NA & NA & NA & NA & NA & NA & NA \\
\hline \multicolumn{12}{|l|}{2011} \\
\hline $18-39$ & 25.2 & 12.2 & $10.8 \dagger$ & 11.8 & 10.4 & 9.6 & 11.1 & 11.1 & 11.5 & 7.5 & 10.1 \\
\hline $40-59$ & 16.3 & $21.3 \dagger$ & 19.2 & 18.2 & 15.2 & 14.9 & 19.9 & 19.6 & 15.2 & 12.7 & 15.2 \\
\hline$\geq 60$ & 21.3 & 19.6 & 16.2 & 18.3 & 14.0 & 14.7 & 14.6 & 17.5 & 14.8 & 12.3 & 14.6 \\
\hline$\%$ change $18-39$ & 76.22 & 5.17 & $-8.47 \dagger$ & -4.84 & 35.06 & 3.23 & -6.72 & 8.82 & 16.16 & -2.60 & 9.78 \\
\hline$\%$ change $40-59$ & -7.39 & $24.56 \dagger$ & 10.34 & -2.15 & 38.18 & 11.19 & 43.17 & 14.62 & 2.01 & 19.81 & 16.92 \\
\hline$\%$ change $\geq 60$ & NA & NA & NA & NA & NA & NA & NA & NA & NA & NA & NA \\
\hline \multicolumn{12}{|l|}{ Obese class II } \\
\hline \multicolumn{12}{|l|}{$2000 / 2001$} \\
\hline $18-39$ & $4.5 \dagger$ & $3.1 \dagger$ & $4.0 \dagger$ & $3.6 \dagger$ & 2.1 & 2.3 & 2.7 & 3.4 & 3.0 & 1.6 & 2.4 \\
\hline $40-59$ & 3.7 & $3.7 \dagger$ & 5.4 & 3.9 & 2.6 & 3.4 & 4.9 & 5.2 & 2.8 & 2.7 & 3.2 \\
\hline$\geq 60$ & NA & NA & NA & NA & NA & NA & NA & NA & NA & NA & NA \\
\hline \multicolumn{12}{|l|}{2011} \\
\hline $18-39$ & $4.7 \dagger$ & - & $3.7 \dagger$ & $6.0 \dagger$ & $1.9 \dagger$ & 3.0 & $3.4 \dagger$ & $4.1 \dagger$ & $2.6 \dagger$ & $1.7 \dagger$ & 2.7 \\
\hline $40-59$ & $5.7 \dagger$ & 一‡ & 8.5 & $7.7 \dagger$ & $2.9 \dagger$ & 5.2 & $4.5 \dagger$ & $3.8 \dagger$ & $6.0 \dagger$ & $3.7 \dagger$ & 4.7 \\
\hline$\geq 60$ & $-\ddagger$ & -‡ & $4.4 \dagger$ & $3.9+$ & 2.5 & 3.5 & $4.2 \dagger$ & $5.4 \dagger$ & 5.1 & 2.1 & 3.3 \\
\hline \multicolumn{12}{|l|}{ Obese class III } \\
\hline \multicolumn{12}{|l|}{$2000 / 2001$} \\
\hline $18-39$ & $1.3 \dagger$ & 一‡ & $2.1 \dagger$ & $1.7 \dagger$ & -† & 1.0 & $1.3 \dagger$ & $1.4 \dagger$ & $0.9 \dagger$ & $0.8 \dagger$ & 1.0 \\
\hline $40-59$ & $1.8 \dagger$ & $1.5 \dagger$ & $1.8 \dagger$ & $2.0 \dagger$ & $0.9 \dagger$ & 1.5 & $2.0 \dagger$ & $1.5 \dagger$ & 1.6 & 1.0 & 1.3 \\
\hline$\geq 60$ & NA & NA & NA & NA & NA & NA & NA & NA & NA & NA & NA \\
\hline \multicolumn{12}{|l|}{2011} \\
\hline $18-39$ & $-\ddagger$ & -‡ & 1.8 & $3.9+$ & $1.1 \dagger$ & $1.5 \dagger$ & 一‡ & $2.5 \dagger$ & $1.2 \dagger$ & $1.6 \dagger$ & 1.5 \\
\hline $40-59$ & - & - & $3.1 \dagger$ & -‡ & $1.3 \dagger$ & 2.0 & $3.0 \dagger$ & $2.2 \dagger$ & $1.8 \dagger$ & $0.9 \dagger$ & 1.8 \\
\hline$\geq 60$ & $-\ddagger$ & -‡ & 一‡ & $2.7 \dagger$ & $1.2 \dagger$ & $1.4 \dagger$ & $2.0 \dagger$ & - & $1.3 \dagger$ & - & 1.4 \\
\hline $\begin{array}{l}\text { Note: BMI = body mas } \\
\text { Data source: Statistics } \\
\text { (compiled by the New } \\
\text { *BMl categories: norm } \\
\text { III (BMI } \geq 40.0 \text { ). } \\
\text { †Data with a coefficie } \\
\text { †Data suppressed be }\end{array}$ & $\begin{array}{l}\text { NA }=n \\
\text { la, Cana } \\
\text { nd and } L \\
18.5-24 \\
\text { riation fro } \\
\text { f extrem }\end{array}$ & $\begin{array}{l}\text { licable. } \\
\text { Communit } \\
\text { lor, Centre } \\
\text { verweight } \\
\text { 6\%-33.3 }\end{array}$ & $\begin{array}{l}\text { Health Sur } \\
\text { for Health I } \\
\text { BMI } 25.0-2 \\
\text { should be } \\
\text { tion. }\end{array}$ & $\begin{array}{l}\text { y, Share } F \\
\text { ormation). } \\
.9) \text {, obese } \\
\text { terpreted }\end{array}$ & $\begin{array}{l}\text { 2000/01 } \\
\mathrm{Ml} \geq 30.0 \text { ) } \\
\text { caution. }\end{array}$ & $\begin{array}{l}-64 \text { years) } \\
\text { ese class }\end{array}$ & $\begin{array}{ll}3,2005 \\
\text { MI } 30.0-\end{array}$ & 9), obese & $\begin{array}{l}\text {-2010, } \\
\text { II (BMI }\end{array}$ & $\begin{array}{l}2011 \text { for } \\
\text {-39.9) an }\end{array}$ & $\begin{array}{l}\text { + years } \\
\text { obese class }\end{array}$ \\
\hline
\end{tabular}

suggested that in some countries, including Canada, the prevalence of obesity will continue to rise at a predicted rate of $4 \%-5 \%$ per year. ${ }^{1}$ Although class I obesity appears to be increasing at a slower rate in Canada, the rate for the higher classes of obesity continues to increase disproportionately, a finding consistent with other studies. ${ }^{32-34}$ These results raise concern at a policy level, because people in these obesity classes are at a much higher risk of developing complex care needs. ${ }^{16,17,35}$ Our findings also show a continuing increase in the regional differences in the prevalence of obesity, results similar to findings reported in other studies. ${ }^{36-38}$ Possible explanations for these variations include population differences in levels of education and income, social class, age demographic, sex, ethnicity, genetic predisposition, and rural versus urban living. However, these relations are not always consistent between or within countries. ., $, 22,39-42^{-1}$

Although rates of increase differ, our prediction analysis estimates a continued increase in obesity in all classes for all provinces from 2013 to 2019. Several prediction studies report continued increases in obesity rates. ${ }^{1,33,43}$ In the United States, it is estimated that by $2030,42 \%$ of the adult population will be obese. $^{33}$ In Australia, by 2025 , most men (83\%) and women (75\%) will be either overweight or obese. ${ }^{43}$ In contrast, some 
countries are experiencing a leveling off of obesity rates, suggesting that country-specific efforts to curb obesity may be having an impact.

In Canada, there is no country-wide coordinated effort to address the adult obesity epidemic. At federal, provincial/territorial and community levels, prevention and health promotion strategies have been established to increase levels of physical activity, improve healthy eating, and reduce the chronic disease burden of obesity. With this common goal in mind, programs across all levels of jurisdiction have been developed, which differ in concept, target audience and implementation techniques. $^{40,44}$ Unfortunately, there is a lack of data available evaluating the effectiveness of these programs. Given the provincial variation in the prevalence of obesity, it may be that some provinces are more successful at implementing strategies aimed at promoting healthy lifestyles and weightmanagement behaviours. Without a complete synthesis of what each province is doing, it is very difficult to know if these differences can be explained by the uptake or implementation of federal, provincial, community-wide or local initiatives. ${ }^{44}$

One potential challenge to country-wide initiatives in Canada stems from the fact that although the publicly funded health system is governed by federal legislation (the Canada Health Act), the actual provision of health services comes under provincial and territorial jurisdiction. The act's principles ensure some consistency among provincial/territorial health systems but cannot influence decisions on health priorities or the allocation of resources at the provincial/territorial levels. These will differ depending on priorities and budgets, and will directly affect the importance given to the health promotion, prevention, management and treatment of obesity. For example, provincial variations may exist in the provision of bariatric surgery to eligible patients for the treatment of morbid obesity, ${ }^{45}$ in the training or lack of training of health professionals in weight management, ${ }^{46,47}$ and in patient access to other types of expertise in weight loss such as dietitians and psychologists. In Canada, a number of resources exist to help health professionals provide evidence-based clinical care to obese patients, such as the clinical practice guidelines on the prevention, management and treatment of obesity, ${ }^{16}$ and the approach of the newly published Canadian Obesity Network 5-A's (ask, assess, advise, agree and assist) approach to obesity management for primary care physicians. ${ }^{48}$ However, the uptake and implementation of these and other weight-management resources have not been studied or evaluated at a provincial level.

\section{Limitations}

The use of BMI as a measure of body fat does not differentiate between lean and fat tissue, and therefore its use in the clinical setting is limited. ${ }^{49,50}$ However, BMI remains useful for the purposes of tracking population trends and comparing obesity prevalence over time. In the current study, BMI in adults aged 18 years and older (with the exception of the 2000/01 CCHS, which was limited to adults aged 20-64 yr) was based on self-reported heights and weights, with the inherent limitation that people tend to underestimate their weight and overestimate their height. ${ }^{51}$ Consequently, this

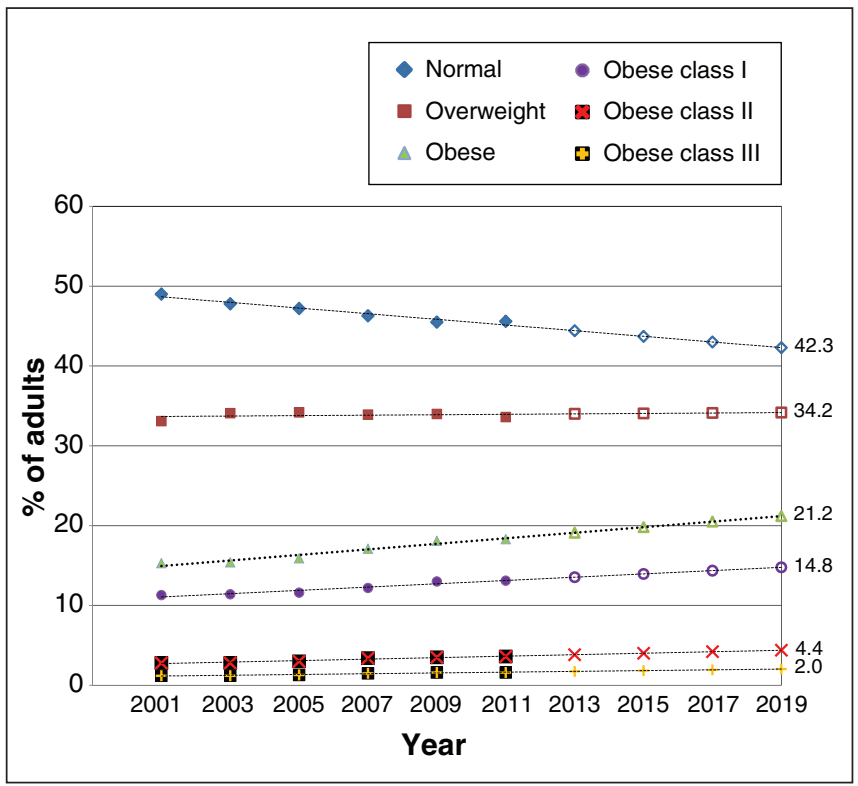

Figure 2: Predictions of future prevalence of adult obesity in Canada, from 2013 to 2019, by weight category: normal (BMI 18.5-24.9), overweight (BMI 25.0-29.9), obese (BMI $\geq 30.0$ ), obese class I (BMI 30.0-34.9), obese class II (BMI 35.0-39.9) and obese class III (BMI $\geq 40.0)$. $\mathrm{BMI}=$ body mass index.

analysis provides conservative estimates of the current and future predicted prevalence of obesity in Canada.

A further limitation is the use of cross-sectional surveys with limited sample size for conducting meaningful subgroup analysis. Larger samples and longitudinal data would provide a more valid and reliable estimate of the prevalence of obesity and in which subgroups it changes over time. Nevertheless, the use of large, nationally representative data sets is a major strength of this study.

Finally, the trend analysis was based on the survey data collected over the last decade, which illustrates increases in a linear fashion. Other Canadian surveys (measured or selfreported) suggest that increases may have occurred at a much faster rate in the 1980s and 1990s, but that, in the past decade, this rate, although still increasing, may have slowed down. ${ }^{52}$ The validity of prediction analyses depends on the assumption that little change will occur during the predicted period. It is unlikely, in our predicted period, that demographic change, new pharmacologic agents or increased access to bariatric surgery will affect the accuracy of these predictions.

\section{Conclusion}

Since 1985 , the prevalence of obesity has increased by $200 \%$ in Canada, with the highest increases occurring in the excessive weight categories. By 2019, it is predicted that about $21 \%$ of the Canadian adult population will be obese. Interprovincial variations show continued variation in the prevalence of obesity. With few exceptions, over the past 10 years, the incidence of obesity increased in all provinces, for both sexes, and across all age groups, but not at the same rate. An improved understanding of why such substantial interprovincial variations exist is necessary, including a focus on 
evaluating existing policies, programs and approaches to the prevention, management and treatment of obesity.

\section{References}

1. Sassi F, Devaux M. OECD obesity update 2012. Paris: Organisation for Economic Co-operation and Development; 2012. Available: www.oecd.org /health/49716427.pdf (accessed 2013 Feb. 21).

2. Katzmarzyk PT, Mason C. Prevalence of class I, II and III obesity in Canada. CMA7 2006;174:156-7.

3. Statistics Canada Canadian Community Health Survey, Share File 2009-2010.

4. Dutton DJ, McLaren L. Explained and unexplained regional variation in Canadian obesity prevalence. Obesity (Silver Spring) 2011;19:1460-8.

5. Shields M, Tjepkema M. Regional differences in obesity. Health Reports 2006;17:61-7. Cat no 82-003.

6. Guh DP, Zhang W, Bansback N, et al. The incidence of co-morbidities related to obesity and overweight. A systematic review and meta-analysis. BMC Public Health 2009;9:88.

7. Luo W, Morrison H, de Groh M, et al. The burden of adult obesity in Canada. Chronic Dis Can 2007;27:135-44.

8. Bray GA. Medical consequences of obesity. 7 Clin Endocrinol Metab 2004;89:2583-9.

9. Kolotkin RL, Meter K, Williams GR. Quality of life and obesity. Obes Rev 2001;2:219-29.

10. McLaren L. Socioeconomic status and obesity. Epidemiol Rev 2007;29:29-48.

11. Puhl RM, Heuer C. The stigma of obesity: a review and update. Obesity (Silver Spring) 2009;17:941-64.

12. Katzmarzyk PT, Ardern CI. Overweight and obesity mortality trends in Canada 1985-2000. Can 7 Public Health 2004;95:16-20.

13. Fontaine KR, Redden DT, Wang C, et al. Years of life lost due to obesity. FAMA 2003;289:187-93.

14. Obesity: preventing and managing the global epidemic. Report of a WHO Consultation. World Health Organ Tech Rep Ser 2000;894:i-xii, 1-253.

15. Canadian guidelines for body weight classifications in adults. Ottawa $(\mathrm{ON})$ : Health Canada; 2003. Cat no H49-179/2003E.

16. Lau DC, Douketis JD, Morrison KM, et al. 2006 Canadian clinical practice guidelines on the management and prevention of obesity in adults and children [summary]. CMAf 2007;176:1103-6. Available: www.cmaj.ca/cgi/content/full /176/8/S1 (accessed 2013 Oct. 29).

17. Twells LK, Bridger T, Knight JC, et al. Obesity predicts primary health care visits: a cohort study. Popul Health Manag 2012;15:29-36.

18. Orpana HM, Berthelot JM, Kaplan MS, et al. BMI and mortality: results from a national longitudinal study of Canadian adults. Obesity (Silver Spring) 2010;18:214-8.

19. Flegal KM, Kit BK, Orpana H, et al. Association of all-cause mortality with overweight and obesity using standardized body mass index categories. A systematic review and meta-analysis. FAMA 2013;309:71-82.

20. Tjepkema M. Adult obesity in Canada: measured height and weight. In: Nutrition: findings from the Canadian Community Health Survey. Ottawa (ON): Statistics Canada. Available: www.aboutmen.ca/application/www.aboutmen.ca /asset/upload/tiny_mce/page/link/Adult-Obesity-in-Canada.pdf (accessed 2012 Nov. 16). Cat. no. 82-620-MWE2005001.

21. Anis AH, Zhang W, Bansback N, et al. Obesity and overweight in Canada: an updated cost-of-illness study. Obes Rev 2010;11:31-40.

22. Obesity in Canada. Ottawa (ON): Public Health Agency of Canada, Canadian Institute for Health Information; 2011.

23. Jia H., Lubetkin E. Trends in quality adjusted life years lost contributed by smoking and obesity. Am 7 Prev Med 2010;38:138-44.

24. Canadian Community Health Survey (CCHS): cycle 1.1: extending the wealth of bealth data in Canada. Ottawa (ON): Statistics Canada; 2001. Available: www.statcan.gc.ca/concepts/health-sante/cchs-escc-info-eng.htm\#back (accessed 2013 Feb. 21)

25. Health promotion survey. Ottawa (ON): Statistics Canada; 1985, 1990. Available: http://www23.statcan.gc.ca/imdb/p2SV.pl? Function=getInstanceList\&SurvId= 3828\&SurvVer $=1 \&$ InstaId $=15750 \&$ SDDS $=3828 \&$ lang $=$ en $\& \mathrm{db}=\mathrm{imdb} \& \mathrm{adm}=8$ \& dis=2 (accessed 2013 Feb. 23).

26. National population health survey - housebold component - longitudinal. Ottawa (ON): Statistics Canada; 1994-2011. Available: http://www23.statcan.gc.ca /imdb/p2SV.pl? Function=getInstanceList $\&$ SurvId $=3225 \&$ SurvVer $=0 \&$ InstaId $=15$ 280\&SDDS $=3225 \&$ lang $=$ en $\&$ db $=$ imdb \&adm $=8 \&$ dis $=2$ (accessed 2013 Feb. 23).

27. Shryock HS, Siegel JS, editors (condensed edition by Stockwell EG). The methods and materials of demography (studies in population). New York: Academic Press; 1976.

28. Census profile. 2011 census. Canada (Code 01) and Canada (Code 01) [table]. Ottawa (ON): Statistics Canada; 2012. Cat no 98-316-XWE. Available: http://www12.statcan.gc.ca/census-recensement/2011/dp$\mathrm{pd} /$ prof/index.cfm? Lang=E (accessed 2013 Feb. 20).

29. Finucane MM, Stevens GA, Cowan MJ, et al. National, regional and global trends in body-mass index since 1980: a systematic analysis of health examination surveys and epidemiological studies with 960 country-years and 9.1 million participants. Lancet 2011;377:557-67.
30. Flegal KM, Carroll MD, Ogden CI, et al. Prevalence and trends in obesity among US adults, 1999-2008. 7AMA 2010;303:235-41.

31. Gotay CC, Katzmarzyk PT, Janssen I, et al. Updating the Canadian obesity maps: an epidemic in progress. Can 7 Public Health 2013;104:e64-8.

32. Sturm R, Hattori A. Morbid obesity rates continue to rise rapidly in the United States [short communication]. Int $\mathcal{F}$ Obes (Lond) 2013;37:889-91.

33. Finkelstein EA, Khavjou OA, Thompson H, et al. Obesity and severe obesity forecasts through 2030. Am 7 Prev Med 2012;42:563-70.

34. Lobstein T, Leach RJ. Foresight. Tackling obesities: future choices - international comparisons of obesity trends, determinants and responses - evidence review. London (UK): Government Office for Science; 2007. Available: www.bis.gov.uk /assets/foresight/docs/obesity/06\%20part\%2001.pdf (accessed 2013 Oct. 29).

35. Withrow D, Alter DA. Economic burden of obesity worldwide: a systematic review of the direct costs of obesity. Obes Rev 2011;12:131-41.

36. Scarborough $\mathrm{P}$, Allender S. The North-South gap in overweight and obesity in England. Br 7 Nutr 2008;100:677-84.

37. Reynolds K, Gu D, Whelton PK, et al.; InterASIA Collaborative Group. Prevalence and risk factors of overweight and obesity in China. Obesity (Silver Spring) 2007; $15: 10-8$.

38. McPherson K, Marsh T, Brown M. Foresight. Tackling obesities: future choices modelling future trends in obesity and their impact on bealth. London (UK): Government Office for Science; 2007. Available: www.bis.gov.uk/assets/foresight /docs/obesity/14.pdf (accessed 2013 Oct. 29).

39. Wang Y, Beydoun MA. The obesity epidemic in the United States - gender, age, socioeconomic, racial/ethnic and geographic characteristics: a systematic review and meta-regression analysis. Epidemiol Rev 2007;29:6-28.

40. Raine K. Overweight and obesity in Canada. A population health perspective. Ottawa (ON): Canadian Institute for Health Information; 2004.

41. McLaren L. Social Class and BMI among Canadian adults: a focus on occupational prestige. Obesity (Silver Spring) 2009;17:290-9.

42. Prättälä R, Sippola R, Lahti-Koski M, et al. Twenty-five year trends in body mass index by education and income in Finland. BMC Public Health 2012;12:936.

43. Haby MM, Markwick A, Peeters A, et al. Future predictions of body mass index and overweight prevalence in Australia, 2005-2025. Health Promot Int 2012; 27:250-60.

44. Prince SA. A population health approach to obesity in Canada - putting the problem back into context. Transdisciplinary Studies in Population Health Series 2009;1:22-3.

45. Padwal RS, Chang HJ, Klarenbach S, et al. Characteristics of the population eligible for and receiving publicly funded bariatric surgery in Canada. Int $\mathcal{F}$ Equity Health 2012;11:54.

46. Kirk SF, Tytus R, Tsuyuki RT, et al. Weight management experiences of overweight and obese Canadian adults: findings from a national survey. Chronic Dis Inj Can 2012;32:63-9.

47. Bleich SN, Bennett WL, Gudzune KA, et al. National survey of US primary care physicians' perspectives about causes of obesity and solutions to improve care. BM7 Open 2012;2:e001871.

48. Vallis M, Piccinini-Vallis H, Sharma A, et al. Modified 5 As: minimal intervention for obesity counseling in primary care. Can Fam Physician 2013;59:27-31.

49. Frankenfield DC, Rowe WA, Cooney RN, et al. Limits of body mass index to detect obesity and predict body composition. Nutrition 2001;17:26-30.

50. Gallagher D, Heymsfield B, Heo M, et al. Healthy percentage body fat ranges: an approach for developing guidelines based on body mass index. Am 7 Clin Nutr 2000;72:694-701

51. Connor Gorber S, Tremblay M, Moher D, et al. A comparison of direct vs. selfreport measures for assessing height, weight and body mass index: a systematic review. Obes Rev 2007;8:307-26.

52. Janssen I. The public health burden of obesity. Can f Diabetes 2013;37:90-6.

Affiliations: From the School of Pharmacy (Twells), Faculty of Medicine (Twells, Gregory, Reddigan, Midodzi), Memorial University, and the Eastern Health Regional Authority (Gregory), St. John's, Newfoundland and Labrador.

Contributors: Laurie Twells conceived the study concept and design and acquired the data. All of the authors contributed to data analysis and interpretation, and drafted and revised the manuscript. All of the authors approved the final version submitted for publication.

Funding: This project was investigator-initiated and received no funding.

Acknowledgements: The researchers used data from Statistics Canada prepared by the Newfoundland and Labrador Centre for Health Information (Dr. Don MacDonald, Vice President of Research and Evaluation); however, the analyses and interpretations presented in the paper do not reflect the views of Statistics Canada.

Supplemental information: For reviewer comments and the original submission of this manuscript, please see www.cmajopen.ca/content/2/1 /E18/suppl/DC1 